\title{
Automatic detection of P, QRS and T patterns in 12 leads ECG signal based on CWT
}

\author{
Maxime Yochum ${ }^{1}$, Charlotte Renaud ${ }^{2}$, Sabir Jacquir ${ }^{1}$ \\ ${ }^{1}$ Laboratoire LE2I UMR CNRS 6306, UniversitÃl' de Bourgogne, 9 avenue Alain Savary, 21078 Dijon, France. (e-mail: sabir.jacquir@u-bourgogne.fr) \\ ${ }^{2}$ Dijon Hospital University, 21000 Dijon cedex, France.
}

\begin{abstract}
In this paper, a new method based on the continuous wavelet transform is described in order to detect the QRS, P and T waves. QRS, P and T waves may be distinguished from noise, baseline drift or irregular heartbeats. The algorithm, described in this paper, has been evaluated using the Computers in Cardiology (CinC) Challenge 2011 database and also applied on the MIT-BIH Arrhythmia database (MITDB). The data from the CinC Challenge 2011 are standard 12 ECG leads recordings with full diagnostic bandwidth compared to the MITDB which only includes two leads for each ECG signal. Firstly, our algorithm is validated using fifty 12 leads ECG samples from the CinC collection. The samples have been chosen in the "acceptable records" list given by Physionet. The detection and the duration delineation of the QRS, P and T waves given by our method are compared to expert physician results. The algorithm shows a sensitivity equal to 0.9987 for the QRS complex, 0.9917 for the T wave and 0.9906 for the $\mathrm{P}$ wave. The accuracy and the Youden index values show that the method is reliable for the QRS, T and P waves detection and delineation. Secondly, our algorithm is applied to the MITDB in order to compare the detection of QRS wave to results of other some works in the literature.
\end{abstract}

Keywords: ECG, P, QRS, T waves, delineation, wavelet transform

\section{Introduction}

The automatic analysis of ECG signal and delineation of P, QRS and T waves are widely studied in the worldwide. This research is motivated by several reasons: The ECG signals are used by physician to diagnose many cardiac diseases and also as part of diagnostic of many non-cardiac pathologies, such as autonomic malfunction, and changes appear in many vascular, respiratory and even psychological dysfunctions. These diseases have a significant economic and social cost in developed countries. The development of tools and systems allowing the detection and the analysis of QRS, T and P waves are relevant to the biomedical industry. In the literature, many methods and tools have been developed for ECG analysis. The QRS, P and $\mathrm{T}$ waves detection is a perfect example, we can cite [1-4]. Some methods are based on sparse derivatives [5], on mathematical model [6], peak detection [7], on nonlinear transform [8], on slope estimation $[9,10]$, on filtering [11], on correlation analysis [12]. Some algorithms are based on the wavelet transform because ECG signals are intrinsically nonstationary. Wavelet transforms [13-18] have been applied to ECG signals to enhance QRS detection, to delineate the ECG feature, and to reduce computation time. An application of cross wavelet transform for ECG pattern analysis and classification has been described in [19]. The shapes of QRS, P and T waves are well known and there is a gold standard in the literature. However, time and frequency parameters of these waves depend on physical and physiological characteristics of people. There may also be some confusions between the frequencies of useful waves and those of noise. Therefore, the algorithm described in this paper is based on the continuous wavelet transform (CWT) which keeps a good frequency resolution. The majority of other ECG delineation processes work with DWT which could lose frequency resolution due to re-sampling at each decomposition. This method has been applied to the Computers in Cardiology Challenge 2011 database [20]. The reason using this database is that it contains 12 leads for each ECG, which is used, by the algorithm presented here, to reinforce the detection of complex response and then avoid some misdetection due to few issues on a lead. The data are standard 12 leads ECG recordings (leads I, II, III, aVR, aVL, aVF, V1, V2, V3, V4, V5, and V6) with full diagnostic bandwidth $(0.05$ through $100 \mathrm{~Hz})$. The leads have been recorded simultaneously for 10 seconds; each lead is sampled at $500 \mathrm{~Hz}$ with 16-bits resolution. In this collection, fifty samples of 12 leads ECG have been used for our tests. The samples have been chosen in the "acceptable records" list given by Physionet. Thus, our algorithm uses all the 12 leads in order to extract QRS, P and T waves from each ECG. This database provides also ECG from different subjects with different pathologies involving irregularities in the ECG. Therefore, our algorithm was tested on several special cases such as arrhythmias and extrasystoles. Then, it was applied to the MITDB in order to compare it with detection results of QRS wave previously published. The MIT-BIH arrhythmia database [21] has 48 annotated records. Each record is about 30 min length with $360 \mathrm{~Hz}$ sampling frequency and includes two leads. The ECG records are compound of a variety of waveforms, artifacts, complex ventricular and conduction abnormalities. Each record is accompanied by an annotation file in which each ECG beat was tagged by expert cardiologists. These labels, referred to as truth 


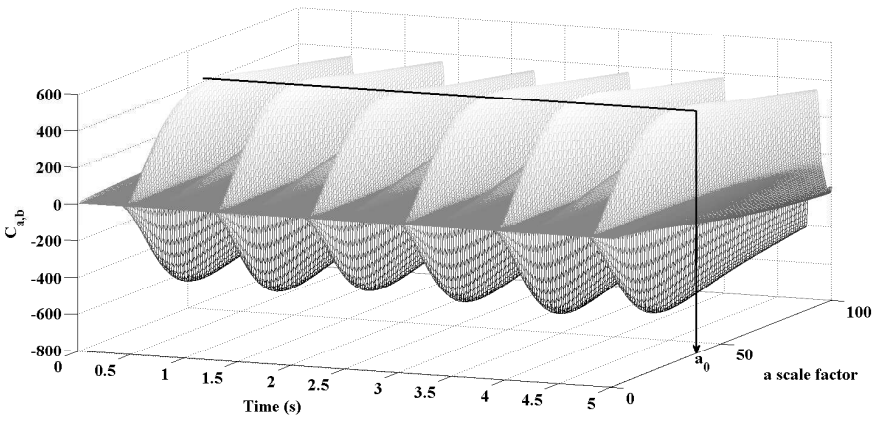

Figure 1: Example of CWT result with Daubechie 3 mother wavelet from $V_{E C G}$ signal plotted in the figure $2 \mathrm{a}$. Scale factors vary from 1 to 100 . The $a_{0}$ scale factor corresponding to the maximum of $C_{a, b}$ is represented by the black arrow.

annotation and are used to evaluate the performance of our algorithm. The algorithm is described in the section 2. It allows a serially detection of the different components of the ECG signal as these components usually follows a decrease in their energies (QRS complexes contain more energy than $\mathrm{T}$ wave, and $\mathrm{T}$ waves contain more energy than $\mathrm{P}$ waves. The results are analyzed and discussed in the section 3 . Finally, the section 4 summarizes our work and gives some perspectives.

\section{Algorithm overview}

The QRS, T and P waves allow evaluating the medical condition of patients. The process consists of detect QRS complex (the largest in amplitude), next the $\mathrm{T}$ wave, and then the $\mathrm{P}$ wave (generally the smallest in amplitude) is deduced. The algorithm based on a CWT uses a known mother wavelet (the Daubechie wavelet) with a varying scale. The scale factor corresponding to the maximum of correlation between the ECG waves and the wavelet is taken as the best one. Using this scale factor, a threshold is determined automatically thanks to a histogram representation. This threshold allows to share correctly the QRS complexes from the ECG lead. Then the algorithm is used a second time to detect $\mathrm{T}$ waves. For a reliable detection, the 12 leads of the ECG are considered in this algorithm. The best area for each wave is determined to be the most common to the 12 leads. In this paper, the algorithm is illustrated using only one among the 12 leads of an ECG.

\subsection{Scale parameter selection}

The CWT is a time frequency analysis method which allows arbitrarily high localization in time of high frequency signal features. The CWT does this by having a varying window width, which is related to the scale of observation. A large selection of localized waveforms can be employed as long as they satisfy predefined mathematical criteria [22, 23]. For its practical implementation, the CWT is computed over a finely discretized time frequency grid. This discretization involves an approximation of the transform integral (i.e. a summation) computed on a discrete grid of scales $a$ and positions $b$ on the time axis. In general, the wavelet transform is approximated in this way over each time step for a range of wavelet scales. To determine each QRS and T wave, we choose the mother wavelet
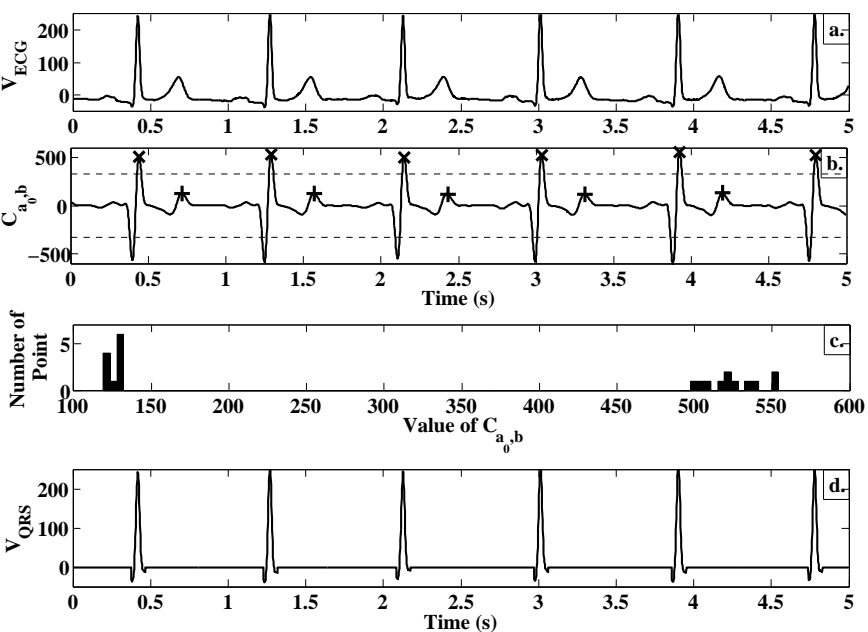

Figure 2: a) $V_{E C G}$ signal example from Physionet collection [20]. b) CWT transformation for the $V_{E C G}$ signal with $a_{0}=38$ corresponding to the maximum of $C_{a, b}$ coefficient. We can also see the representation of the difference between maxima of $C_{a_{0}, b}$ coefficients during QRS complexes (cross) and maxima $C_{a_{0}, b}$ coefficients during $\mathrm{T}$ complexes (plus) which allows the localisation of QRS complexes. Both dashed lines are the $h$ threshold representation for positive and negative parts computed with the equation 2. c) Histogram of local maxima values. We distinguish a bimodal distribution. d) $V_{Q R S}$ result example with $m_{O R S}$ mask applied on the $V_{E C G}$ signal in (a). A reliable delineation of QRS complexes is observed.

which gives the best results according to the reliability indexes presented in section 3.3.1 : Daubechie 3. The use of Daubechie wavelet is also correlated with other studies for ECG analysis $[24,25]$. The CWT is applied between the ECG lead named $V_{E C G}$ and a wavelet $\psi$ (Daubechie 3 wavelet) undergoing the scale factor $a$. Such as:

$$
C_{a, b}\left(V_{E C G}(t), \psi(t)\right)=\int_{-\infty}^{\infty} V_{E C G}(t) \frac{1}{\sqrt{a}} \psi\left(\frac{t-b}{a}\right) d t,
$$

The scale factors have been chosen to go from 1 to 100 by step of 1 . This range allows the analysis of many different ECG lead complex sizes. In the figure 1 , a continuous wavelet transform result with the above parameters is shown. The used $V_{E C G}$ signal corresponds to that one in the figure 2(a). In order to find the scale factor $a$ which has a maximum of correlation with the QRS complex in $V_{E C G}$, we need to estimate which scale factor corresponds to the maximal value of the CWT coefficients matrix, named $C_{a, b}$. The best scale factor is called $a_{0}$.

\subsection{Mask selection}

A temporal mask in the wavelet domain is now computed using the $C_{a, b}$ coefficients with $a_{0}$ scale factor. It allows the discrimination of QRS complexes in the ECG lead. In the figure 2(b), an example of a continuous wavelet transform applied on a $V_{E C G}$ is shown. Only five seconds out of ten have been depicted for a better readability. In this case, the $a_{0}$ scale factor was 38. It has been noticed that $C_{a_{0}, b}$ values corresponding to QRS complexes are more important than $C_{a_{0}, b}$ values corresponding to $\mathrm{T}$ or $\mathrm{P}$ waves, which is not always the case in $V_{E C G}$ as we will see in the next section. Using this fact, QRS 
complexes can be distinguished from $V_{E C G}$. A threshold is created automatically using the local maxima which is used to find maxima coefficients during QRS and maxima coefficients during $\mathrm{T}$ waves. In the figure 2(b), those maxima are represented by crosses (during QRS) and plus (during T). In the figure 2(c), the histogram of those maxima is plotted and shows a bimodal distribution. The threshold sharing correctly QRS complexes from $V_{E C G}$ signal is located between these two distributions. To automatically find the threshold, the centroid of these points is computed in the histogram:

$$
h=\frac{\sum_{i=1}^{n} x_{i} y_{i}}{\sum_{i=1}^{n} y_{i}},
$$

where $h$ is the centroid value, $x_{i}$ are the $C_{a_{0}, b}$ values, $y_{i}$ are the distribution values of the $C_{a_{0}, b}$ coefficients and $n$ represents the histogram range. Then, once the threshold is computed, a mask is created using $\left|C_{a_{0}, b}\right|$ absolute coefficients values and the threshold $h$. The mask is equal to 1 if $\left|C_{a_{0}, b}\right|$ are above the threshold, which corresponds to the QRS complexes and the mask is equal to 0 if $\left|C_{a_{0}, b}\right|$ are below the threshold, corresponding to the $\mathrm{T}$ or $\mathrm{P}$ wave parts. Therefore,

$$
m_{p}= \begin{cases}1 & \text { if }\left|C_{a_{0}, b}\right| \geq h, \\ 0 & \text { if }\left|C_{a_{0}, b}\right|<h,\end{cases}
$$

where $m_{p}$ is the preliminary mask which needs to be adjusted. Indeed, seeing the $C_{a_{0}, b}$ coefficient values in the figure 2(b) with the threshold (the dashed lines), one notices that during QRS complexes, the mask $m_{p}$ has some glitches between the minimum and the maximum of $C_{a_{0}, b}$ coefficients. To correct that, an erosion algorithm (a mathematical morphology operation [26]) is applied to the preliminary mask vector and gives the final mask named $m_{Q R S}$.

\subsection{QRS complexes delineation}

The last step to delineate QRS complexes is to multiply the mask $m_{Q R S}$ previously created to $V_{E C G}$ signal.

$$
V_{Q R S}(t)=V_{E C G}(t) \cdot m_{Q R S}(t),
$$

An example of $V_{Q R S}$ is shown in figure 2(d) which corresponds to the $V_{E C G}$ signal in the figure 2(a). As we can see, QRS complexes are well delineated from $V_{E C G}$ signal.

\subsection{T and $P$ waves delineation}

After QRS complexes delineation is done, QRS complexes are removed by subtracting $V_{Q R S}$ from the $V_{E C G}$ signal.

$$
V_{E C G}^{\prime}(t)=V_{E C G}(t)-V_{Q R S}(t)
$$

An example is shown in the figure 3(a). Then, the previous algorithm (refer to sections 2.1 and 2.2) is applied once again. When CWT is computing again, to detect the T wave, the parts of the signal, which have already been detected as a QRS complex, are not considered anymore. Therefore, when the second CWT is applied, fast transient added by the equation (5) are not
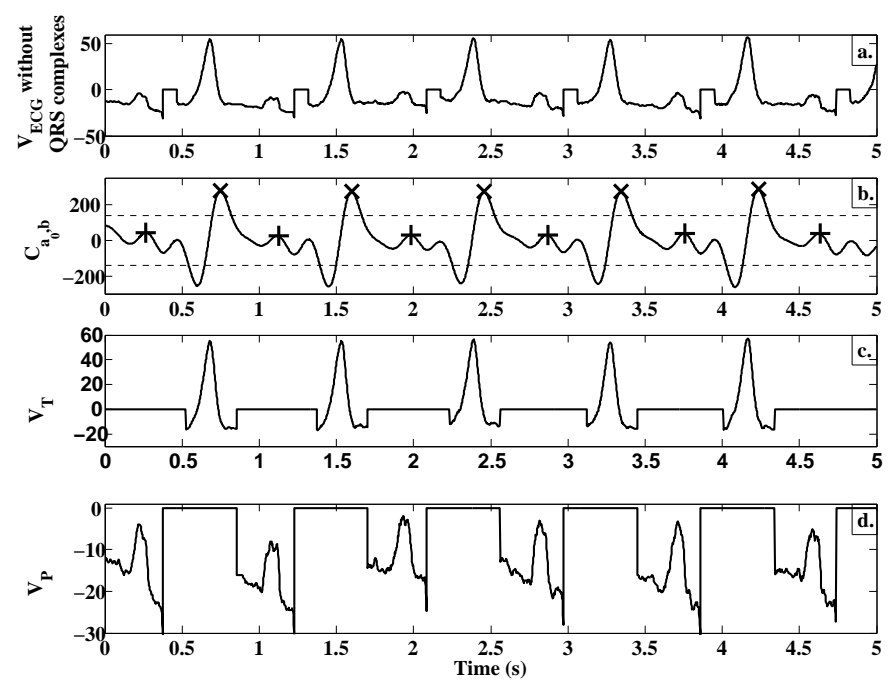

Figure 3: a) $V_{E C G}^{\prime}$ signal without QRS complexes corresponding to examples in figures 2. b) $C_{a_{1}, b}$ coefficients from CWT with $a_{0}$ scale factor. Both dashed lines are the $h_{1}$ threshold representation for positive and negative parts computed from the equation 2. c) $V_{T}$ delineation result example. d) $V_{P}$ delineation result example. A reliable delineation of $\mathrm{T}$ and $\mathrm{P}$ waves is observed.
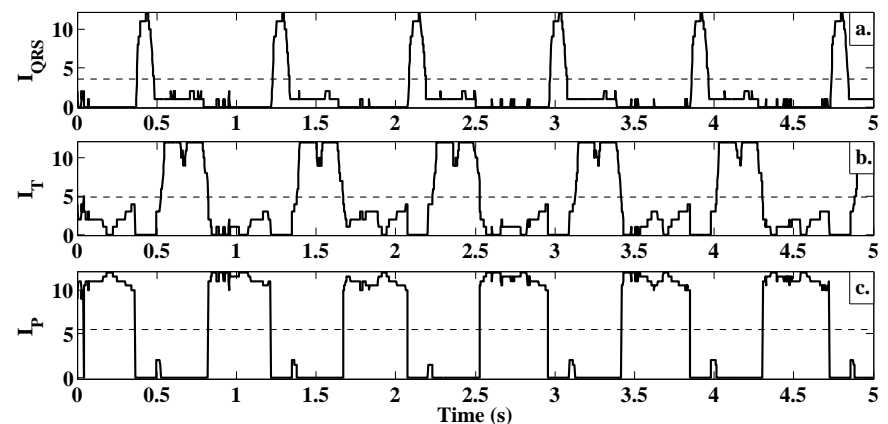

Figure 4: Examples of results of reliability indexes in a) for the QRS complexes, in $\mathrm{b}$ ) for the $\mathrm{T}$ waves and in $\mathrm{c}$ ) for the $\mathrm{P}$ waves. The threshold is plotted with dashed lines for each wave as the mean of the reliability index.

considered. A new scale factor $a_{1}$ is computed from the equation (1) with $V_{E C G}^{\prime}$ signal. In the figure $3(\mathrm{~b})$, we see $C_{a_{1}, b}$ coefficients corresponding to the CWT of the $V_{E C G}^{\prime}$ signal thanks to a new automatically computed threshold value $h$. The local maxima are represented by crosses (during $\mathrm{T}$ waves) and plus (during $\mathrm{P}$ waves). Thanks to those local maxima, the threshold has been computed and plotted with the dashed line. Then, the mask $m_{T}$ is determined and used to find the T waves $V_{T}$ with:

$$
V_{T}(t)=V_{E C G}^{\prime}(t) \cdot m_{T}(t)
$$

An example of $V_{T}$ is shown in the figure 3(c) which corresponds to the $V_{E C G}$ signal in the figure 2(a). As we can see, $\mathrm{T}$ waves are well delineated from $V_{E C G}$ signal. The $\mathrm{P}$ waves are detected as the rest of the $V_{E C G}$ signal without QRS complexes and T waves. In addition, the smallest remaining segments are removed. An example is shown in the figure 3(d).

\subsection{Combination of 12 leads ECG}

Our algorithm allows the localization and delineation of each QRS, T and P waves in an ECG lead. However, it can append 
that one or few leads have some disturbances in it. These disturbances can be noise, particular heart beats inducing change in the ECG scheme (extrasystole for instance) or even a missing lead, which obviously leads to the impossibility to detect anything. To improve the detection of the algorithm, several leads are considered and the answer of each lead is used to compute a reliability index which reinforces the choice of detected area. The database from Physionet [20], used in this work, gives 12 leads ECGs which means that each patient has several ECG electrodes on his skin. That leads to have 12 recorded ECGs at the same time (synchronously). In cardiac diagnostic tests, it is common to have several leads ECGs records. In order to render the localization of QRS, T and $\mathrm{P}$ waves more robust, the 12 leads ECGs are used and the localization results are combined. Even if few leads are unusable, the merging of the 12 leads results could determine the best area of each wave kind. To use the 12 leads, the masks $m_{Q R S_{k}}, m_{T_{k}}$ and $m_{P_{k}}$ are created from each lead (where $k=[1,12], k \in \mathbb{N}$ ). Those masks allow the localization of the QRS, T and P waves into each lead using the algorithm described previously (refer to section 2.1 to 2.4). Therefore, if the localization result is common to the 12 leads, then there is a very strong probability that the waves are well detected. In the other hand, if there are only few localizations among the 12 leads, then there is a low probability that the localization result is true. A reliability index of each QRS complex, $\mathrm{P}$ or $\mathrm{T}$ wave is then computed as the sum of the 12 leads masks:

$$
I_{w}(t)=\sum_{k=1}^{12} m_{w_{k}}(t)
$$

where $w$ is QRS, T or P. Therefore, $I_{Q R S}$ is the reliability index for QRS complexes, $I_{T}$ is the reliability index for $\mathrm{T}$ waves and $I_{P}$ is the reliability index for $\mathrm{P}$ waves. The closest this index is to 12 , the more likely the detected localization is true. In the opposite case, more the index is close to 0 , less the probability that the complex localization is true. In the figure 4, the results of reliability indexes are shown (in (a) for the QRS complexes, in (b) for the $\mathrm{T}$ waves and in (c) for the $\mathrm{P}$ waves).

An automatic threshold can be computed for each wave to be the mean of the reliability index. In the figure 4, these thresholds are marked by dashed lines. The localization of a complex is considered true if the value of the reliability index is above the threshold otherwise it is considered false.

The mean computation processing time of this algorithm is $0.81 \pm 0.02 \mathrm{~s}$ for $10 \mathrm{~s}$ long ECG and on the 12 leads (500 Samples/s on each lead). The computation time of the algorithm depends on several factors, as example the ECG waves morphological variability, the sampling frequency, the operating system, the computation power of the computer, the memory and the CPU. We give some values of computation time from litterature as example in table 1. Our algorithm is implemented with Matlab, an implementation in $\mathrm{C}$ language could lead to a decrease of this computation time by a factor 10 and even more if a parallelization process is used. In this case, it could be implemented in a real time application like a smartphone.
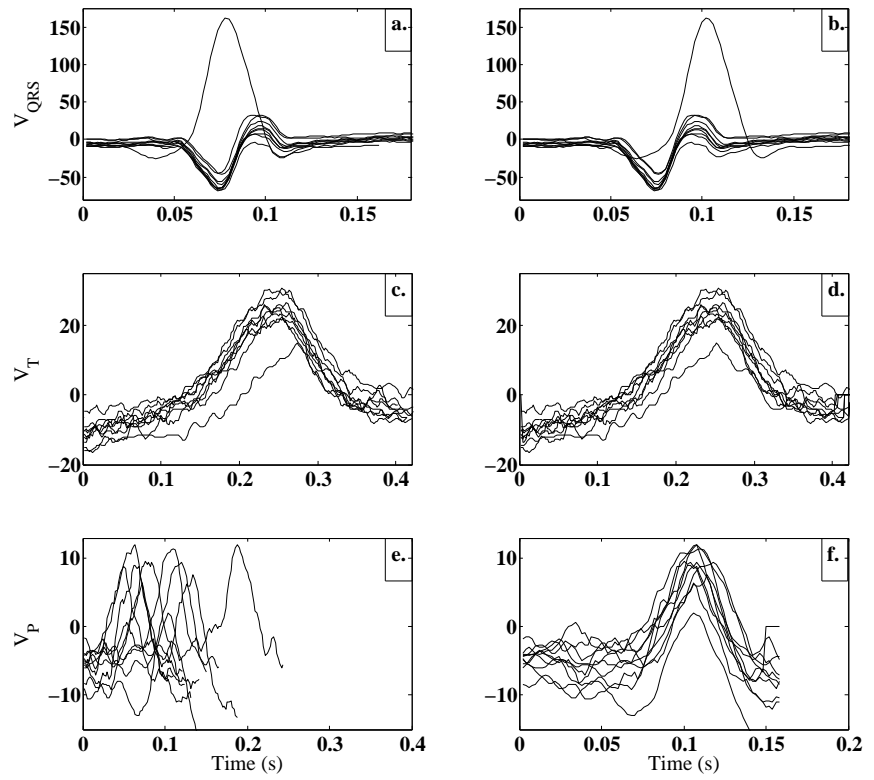

Figure 5: In this figure, examples of realignment are shown for QRS complexes (in (a) and (b)), T waves (in (c) and (d)) and $\mathrm{P}$ waves (in (e) and (f)). In the subfigures (a), (c) and (e), the original waves are plotted. We see in (e) that the $\mathrm{P}$ waves are desynchronised. In the subfigures (b), (d) and (f), the waves are realigned. It is particularly visible for the $\mathrm{P}$ waves in (f).

\subsection{Realignment of $P, Q R S, T$ waves}

As shown in figure 5, the example of the ECG signal contains eleven complete cardiac cycles. A realignment of each kind of wave is performed. The interest of this is to have a superimposed view of the ECG complexes allowing a better characterization of ECG information for physician. In figure 5, examples of layered waves are shown for QRS in (a), T in (d) and P in (g). The realignment is performed for $i$ equal to 2 to 11 (Obviously, the realignment is not made for $i=1$ ) and $w$ is QRS, T or P.

$$
\Gamma_{V_{w_{1 i}}}(n)=\sum_{x=-N}^{N} V_{w_{1}}(x) V_{w_{i}}(x+n T e)
$$

where $x$ is the sample value of the signal, $T e$ is the sample rate and $n=[-N, N], n \in \mathbb{Z}$ where $N$ is the number of samples in $V_{w_{i}}$. The maximum of $\Gamma_{V_{w_{1 i}}}$ gives us the $n$ value corresponding to the delay or the advance of the wave $V_{w_{i}}$ from the first one $V_{w_{1}}$. Then, we can perform the realignment by shifting the $V_{w_{i}}$ wave to $n$ samples. In the figure 5, examples of layered waves with the realignment are shown for QRS in 5(b), T in 5(d) and $P$ in 5(f). We see, in particular for the $P$ waves, that the realignment is well performed.

\section{Results}

\subsection{Application to different cases}

An example of results is shown in the figure 6. Only the first two seconds are displayed for a better readability. A good discrimination of each QRS complex (black lines), T wave (dark gray lines) and $\mathrm{P}$ wave (pale gray lines) is observed. In the 
Table 1: Comparison of computation time.

\begin{tabular}{|c|c|c|c|}
\hline Works & ECG data & Waves studied & Processing time (s) \\
\hline Li et al. [14] & 10 min, 2 leads & P-QRS-T & 60 \\
\hline Madeiro et al. [27] & 15 min, 2 leads & QRS & 4.52 \\
\hline Yeh et Wag [28] & 10 min, 2 leads & QRS & 30 \\
\hline Manikandan et al. [29] & 15 min, 1 lead & R & 2.24 \\
\hline Our method & 10 min, 12 leads & P-QRS-T & 48.6 \\
\hline
\end{tabular}
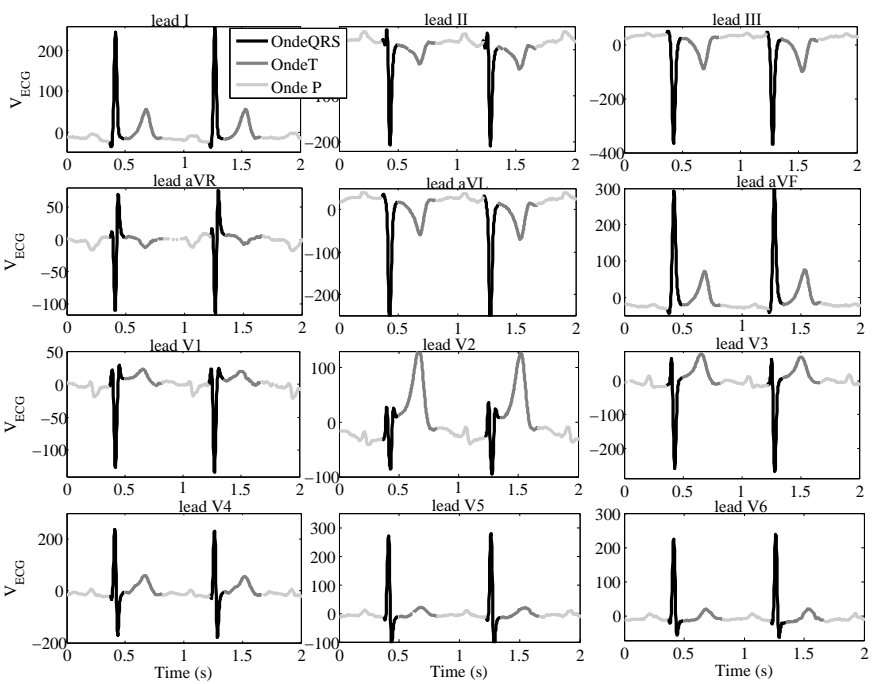

Figure 6: Example of results with one of the 12 leads ECGs from the Computers in Cardiology Challenge 2011 database [20]. We can observe a good discrimination of each QRS complex (black lines), T wave (dark gray lines) and $\mathrm{P}$ wave (pale gray lines).

figure 6, we note the algorithm is still able to detect particular waves. For example, in the lead V2, the QRS complexes are smaller in amplitude than the T ones. In this case, standard thresholding algorithms are not able to discriminate correctly QRS complexes and T waves, they could be mixed up. In figure 7, four different examples are given to show several particular cases where the determination of QRS, T and $\mathrm{P}$ waves could be difficult. In the subfigure 7(a), the SNR ratio of the ECG is really poor, nevertheless the different waves are well detected by our algorithm without any preprocessing (denoising [30], amplitude enhancement [31],...). In the subfigure 7(b), the $T$ waves are higher than the QRS ones. That could lead to mix up those two if a thresholding technique is used directly on ECG signal. However, in our case the thresholding is done in the wavelet domain. The result shows that the correct complexes are detected. It is possible that the baseline of an ECG is not stable. It is the case in the subfigure 7(c). Despite that, the algorithm shares correctly each wave without low frequencies filtering. In the subfigure $7(\mathrm{~d})$, we can see that all waves are in the same range of amplitude which could lead to mix them. However, the robustness of our algorithm prevents this and the results are therefore not affected. Among the samples from the Physionet collection, some ECGs have particular shapes. Our algorithm is able to run even with unusable leads. A lead could be unusable in several condition. For instances, when a lead has got an is-
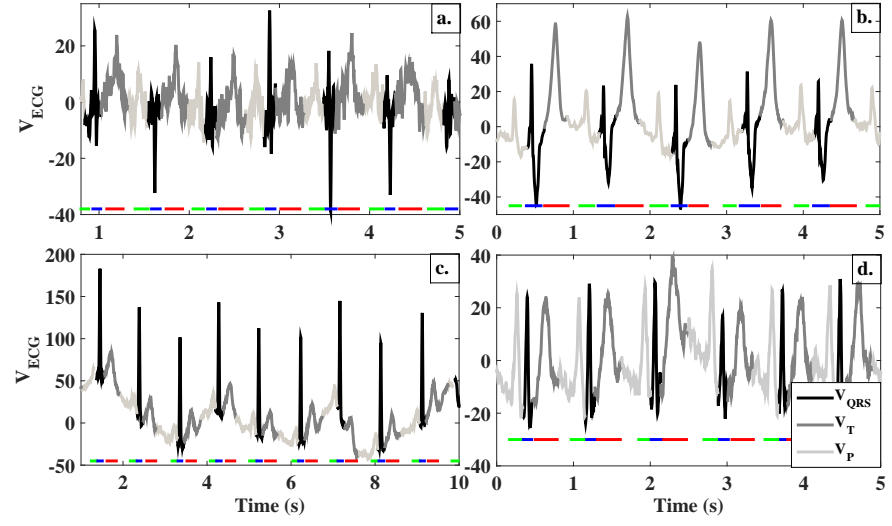

Figure 7: Example of results with some cases where the ECG complexes are usually difficult to distinguish. a) the ECG has got a poor SNR ratio. We see that the algorithm is able to discriminate each wave without filtering. b) the T waves are higher than the QRS ones. These two waves could be mixed up if we use a thresholding technique directly on the ECG signal. However, in our case the thresholding is done in the wavelet domain. c) The baseline of the ECG is not stable. Despite that, the algorithm share correctly each wave. d) All waves are in the same range of amplitude and the results are not affected. QRS (black lines), $\mathrm{T}$ (dark gray lines) and $\mathrm{P}$ (pale gray lines). The tags of the doctor have been added with color lines: QRS (blue lines), T (red lines) and P (green lines)

sue with the associated circuitry or the connection between the electrode and the skin is cut off or if the lead is very noisy. In these particular cases, as our algorithm works on the 12 leads, it gives better results. The reliability index, computed from equation 7, allows compensating the bad results of few leads defaults by the results of other leads, more numerous, which give good detection. In figure 8, examples of extrasystoles which are often present in ECG are shown. The extrasystole is ventricular premature beats which is not initiated at the sinoatrial node [32]. That induces, in the ECG, a perturbation which can invert the QRS complex and T wave and can overlap the $\mathrm{P}$ wave which vanishes from the ECG. As we see in figure 8, these disturbances are correctly detected by our algorithm (see 8(a) and 8(b)). In 8(c) an arrhythmia signal is shown where the $P$ wave is lost in atrial fibrillation (tremulous ECG). However, our algorithm delineates correctly the $\mathrm{P}$ wave potential localization.

\subsection{Improvement of $Q R S, T$ and $P$ waves delineation}

To find fiducial points in the ECG, we add a complementary step in the delineation process. The determination of the various intervals (e.g. RR, PP and QT intervals) and segment (QT) requires the knowledge of boundary locations (onsets and offsets) of the P, QRS, and T waves. Fiducial points detec- 


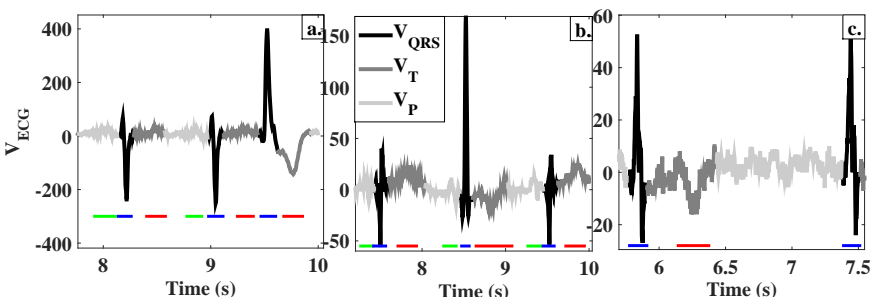

Figure 8: In a) and b), two examples of extrasystole: The QRS complex and T wave are inverted and have different magnitudes. We note that our algorithm is able to detect each complex despite of the extrasystoles influence on the ECG. On the left plot, we see that the QRS complex overlaps the $\mathrm{P}$ wave which is no more present, that is well detected by our algorithm. In c) an arrhythmia signal is shown where it is difficult to determine the $\mathrm{P}$ wave. In this case, our algorithm delineates a location where $\mathrm{P}$ wave should be however a physician is more able to determine if the $\mathrm{P}$ wave is present or not. The tags of the doctor have been added with color lines: QRS (blue lines), T (red lines) and P (green lines)
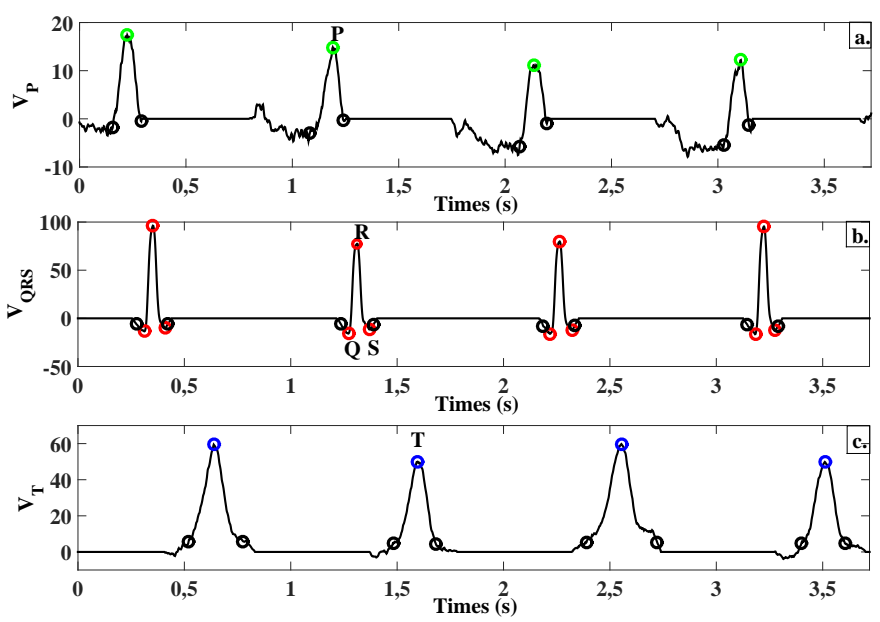

Figure 9: Samples of P (upper item, green circles), QRS (center item, red circles) and $\mathrm{T}$ (bottom item, blue circles) waves with the onsets and the ends (marked by black circles).

tion is complex due to different factors: Non-linearity, nonstationarity, missing beats and noise in the ECG signal. The detection process of fiducial points need morphology, geometry, and rhythm information as we can found in some methods $[4,18,33]$. To complete our method, the position of fiducal points is computed at $90 \%$ from the maximal amplitude of $\mathrm{P}$, $\mathrm{R}$ and $\mathrm{T}$ waves after the step described in sections from 2.1 to 2.5 (see Fig .9). Thus, the boundaries (the onsets and offsets) of the P, QRS, and T waves are defined (see Fig .10). We choose the threshold of $90 \%$ referring to physiologically admitted definition that the action potential duration (APD) is computed at $90 \%$ of the repolarization phasis of the monophasic action potential. Some works show the relationship between the APD and some ECG waves. [34, 35]. The action potential duration is computed from $90 \%$ of its amplitude.

\subsection{Comparison and discussion of results}

\subsubsection{With physician expert identification}

The delineation results given by our algorithm are compared with the results from a physician. The interest is to have the

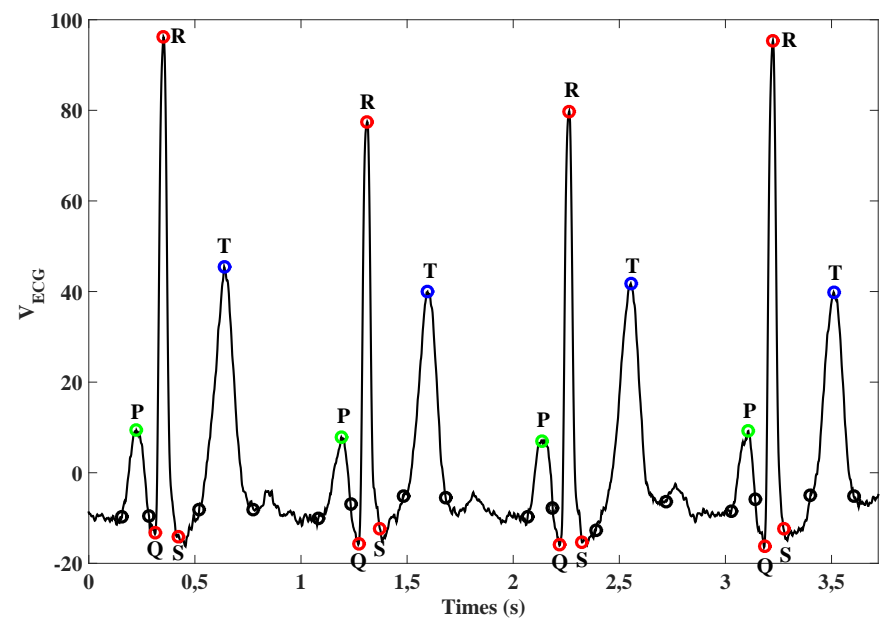

Figure 10: Example of delineation of $\mathrm{P}$ (green circles), QRS (red circles) and $\mathrm{T}$ (blue circles) waves in the ECG (the onsets and the ends of each wave are marked by black circles).

beginning and the end of each ECG wave from an expert as a true result. The physician chooses one lead from the 12 leads ECG and determines the onset and offset of each QRS, P or T wave of this lead. These data are then used as true moments and delineation points of ECG waves. Thanks to our algorithm and the physician, two results for 12 ECG leads are obtained: the real moments $D_{w}(n)$ of QRS, T and P in the ECG tagged by the physician and the results $A_{w}(n)$ given by our algorithm. $A_{w}(n)$ and $D_{w}(n)$ are equal to 1 if the QRS, T or P wave is detected and 0 (noted $\overline{A_{w}(n)}$ and $\left.\overline{D_{w}(n)}\right)$ if is not, they are therefore logical vectors. For each $A_{w}(n)$ and $D_{w}(n)$ pair, the coverage rate $S e_{w}$ is computed ( $w$ denotes QRS, T and P waves.). This coverage is determined as a logical AND between $A_{w}(n)$ and $D_{w}(n)$ divided by $D_{w}(n)$ as shown in the eq.(13). The coverage gives the common result between the algorithm and the physician determination. This coverage is well known as the sensitivity which is computed thanks to TP (True Positive), TN (True Negative), FP (False Positive) and FN (False Negative) where

$$
\begin{aligned}
& T P_{w}=\sum_{n=0}^{N} A_{w}(n) \cdot D_{w}(n), \\
& T N_{w}=\sum_{n=0}^{N} \overline{A_{w}(n)} \cdot \overline{D_{w}(n)}, \\
& F P_{w}=\sum_{n=0}^{N} A_{w}(n) \cdot \overline{D_{w}(n)}, \\
& F N_{w}=\sum_{n=0}^{N} \overline{A_{w}(n)} \cdot D_{w}(n) .
\end{aligned}
$$

Those values are then scalars. Therefore, the sensitivity is computed as:

$$
S e_{w}=\frac{T P_{w}}{T P_{w}+F N_{w}} .
$$


Table 2: Mean values of sensitivity, specificity, Youden index, accuracy, positive predictive value from the fifty ECG samples (CinC Challenge 2011, Physionet database)

\begin{tabular}{|c|c|c|c|c|c|}
\hline Waves & $S e(\%)$ & $S p(\%)$ & $Y(\%)$ & $A c c(\%)$ & $P+(\%)$ \\
\hline QRS & 99.87 & 98.42 & 98.29 & 98.64 & 91.75 \\
\hline T & 99.17 & 93.21 & 91.38 & 94.83 & 84.46 \\
\hline P & 99.06 & 91.21 & 90.27 & 92.44 & 83.22 \\
\hline
\end{tabular}

$S e_{w}$ shows the ability of our algorithm to give the same results as the physician. For each ECG, the specificity has been determined.

$$
S p_{w}=\frac{T N_{w}}{T N_{w}+F P_{w}} .
$$

From the equations (9) to (14), the Youden $Y_{w}$ and the accuracy $A c c_{w}$ indexes can be defined:

$$
\begin{gathered}
Y_{w}=S e_{w}+S p_{w}-1, \\
A c c_{w}=\frac{T P_{w}+T N_{w}}{T P_{w}+T N_{w}+F P_{w}+F N_{w}} .
\end{gathered}
$$

In order to compare our results with some results published in literature, an additional metric is introduced a $P+$, positive predictive value, defined by:

$$
P+_{w}=\frac{T P_{w}}{T P_{w}+F P_{w}} .
$$

Sensitivity, specificity, Youden index, accuracy and predictive values have been performed on the database and the mean values of these indexes are given in the table 2. These indexes show a good ability of our algorithm to detect ECG complexes. The specificity for $\mathrm{T}$ and $\mathrm{P}$ complexes are a little bit lower. This is due to the fact that our algorithm delineates sometimes a larger area than the physician which induces more important TN values. To improve our results, some other processing $[39,40]$ could be done in order to better match durations of $\mathrm{T}$ and $\mathrm{P}$ waves delinetated by our algorithm to a physician delineation.

\subsubsection{Validation of our algorithm with a MIT database (MITDB)}

The Table 3 shows results of some previously published works. These results represent only the $R$ detection obtained from MIT database. In this table, we see that the sensitivity $S e$ and the predictivity $P+$ values, computed from our algorithm in the QRS delineation are very close to the literature. In all cited works of the table 3, the detection of the QRS is compared to the annotations given in the MITDB that includes 48 records of two ECG leads (30 min each). We see that the total number of beats ( $R$ positions) is variable (see a,b,c,d,e,f,g indicate the different total number of beats used in the different works cited in the Table 3). In some works labeled by (a) in the Table 3, the number of true beats (TP) detection is not reported $(\mathrm{N} / \mathrm{R})$. The total number of beats of the 48 records in (a) is higher than in the MITDB: 109963 (+48 beats if the first line of each record is included). The work labelled $(b)$ in the
Table 3 has the exact beats number in the MITDB. The record 207 has a data segment showing non identifiable patterns. Note that these intervals are annotated by exclamation marks (!) in the MITDB. If these parts are excluded to the record 207, the total number of beats corresponds to the works labelled $(c)$ in the Table 3 (109963 - 472 beats with a variation of \pm 3 beats). In the works labelled $(d, e, f)$, the total number of beats is lower than the MITDB due to some selection criteria applied to some of the 48 records. As example, we find details in [3], "some segments with saturation are replaced by those of the other lead (eg, record 116: $1354.2 \mathrm{~s}$ to $1361.9 \mathrm{~s}$ )". This kind of data selection improves probably the detection result as we can see in the table 3 . In this context, considering only two leads, if signal of one lead is not usable, the result of QRS detection could be questionable. Then, our algorithm based on the analysis of the twelve ECG leads can improve the result of QRS detection because a large choice of leads (12 leads) can be used. Although, our method uses only two leads, detection results can be improved, in particular when a lead has failed.

\section{Conclusion}

In this paper, a new QRS, T and P waves delineation method is proposed. This algorithm is performed with the CWT and the histogram representation to determine a mask selection of the QRS, T and P waves. It has been tested with the Computers in Cardiology Challenge 2011 database and the MIT-BIH Arrhythmias database. It can simultaneously delineate the QRS, $\mathrm{T}$ and $\mathrm{P}$ patterns on each ECG lead. The results show a localization of the QRS, T and P waves into an ECG signal which is quantified through different indexes as sensitivity and accuracy. Our algorithm has been tested on different particular cases where it is difficult to determine rigorously each wave position. It is the case for noisy signals, baseline and magnitude issues and disturbances (extrasystole and arrhythmia). In order to improve our algorithm, a delineation step allowing the boundary locations (onsets and offsets) of P, QRS, and T waves is proposed. We also show that the fact of using a 12 leads ECG allows a good determination of complexes location for those particular cases. In our validation step, the computation of the quality indexes (from TP, TN, FP and FN) are based on the determination of onsets and offsets for each complex in the ECG from a physician. Therefore, those results depend on the subjectivity of the physician. However, the determination of complexes is well known from physician and their localization should not be so much affected from a physician to another. The sensitivity and the predictivty values of the QRS detection are close to previously published results in the case of the MITDB database.

\section{Reference}

[1] J. Pan, W. Tompkins, A real time qrs detection algorithm, IEEE Trans. Biomed. Eng. 32 (3) (1985) 230-236.

[2] O. Pahlm, L. SÃúrnmo, Software qrs detection in ambulatory monitoringâĂŤa review, Med. Biol. Eng. Comput. 22 (4) (1984) 289-297. 
Table 3: QRS detection results from the MITDB database in the literature. N/R not reported. a, b, c, d, e, f indicate the different total number of beats used in the different works cited in this table.

\begin{tabular}{|c|c|c|c|c|c|c|c|}
\hline QRS detector & Total (Beats) & TP (Beats) & FP (Beats) & FN (Beats) & Se \% & $P^{+} \%$ & $\%$ error \\
\hline Karimipour-Homaeinezhad [18] & $116137^{a}$ & $\mathrm{~N} / \mathrm{R}$ & 308 & 192 & 99.81 & 99.7 & 0.49 \\
\hline Pan-Tompkins [1] & $116137^{a}$ & $\mathrm{~N} / \mathrm{R}$ & 507 & 277 & 99.75 & 99.53 & 0.675 \\
\hline Li et al. [14] & $116137^{a}$ & $\mathrm{~N} / \mathrm{R}$ & 65 & 112 & 99.89 & 99.94 & 0.15 \\
\hline Yeh et al. [33] & $116137^{a}$ & $\mathrm{~N} / \mathrm{R}$ & 58 & 166 & $\mathrm{~N} / \mathrm{R}$ & $\mathrm{N} / \mathrm{R}$ & 0.19 \\
\hline Poli et al. [36] & $109963^{b}$ & 109522 & 545 & 441 & 99.6 & 99.5 & 0.9 \\
\hline Madeiro et al. [37] & $109495^{c}$ & 108568 & 856 & 928 & 99.15 & 99.18 & 1.69 \\
\hline Zidelman et al. [15] & $109494^{c}$ & 109101 & 193 & 393 & 99.64 & 99.82 & 0.54 \\
\hline Our work & $109491^{c}$ & 109331 & 574 & 160 & 99.85 & 99.48 & 0.67 \\
\hline Martínez et al. [16] & $109428^{d}$ & 109208 & 153 & 220 & 99.80 & 99.86 & 0.34 \\
\hline Hamilton-Tompkins [38] & $109267^{e}$ & 108927 & 248 & 340 & 99.69 & 99.77 & 0.54 \\
\hline Zidelman et al. [3] & $108494^{f}$ & 108323 & 97 & 171 & 99.84 & 99.91 & 0.25 \\
\hline
\end{tabular}

[3] Z. Zidelmal, A. Amirou, D. Ould-Abdeslam, A. Moukadem, A. Dieterlen, Qrs detection using s-transform and shannon energy, Comp. Methods Prog. Biomed. 116 (1) (2014) 1-9.

[4] R. Tafreshi, A. Jaleel, J. Lim, L. Tafreshi, Automated analysis of ecg waveforms with atypical qrs complex morphologies, Biomed. Sig. Proc. and Cont. 10 (2014) 41-49.

[5] X. Ning, I. W. Selesnick, Ecg enhancement and qrs detection based on sparse derivatives, Biomedical Signal Processing and Control 8 (6) (2013) 713-723.

[6] J. P. Madeiro, W. B. Nicolson, P. C. Cortez, J. A. Marques, C. R. VázquezSeisdedos, N. Elangovan, G. A. Ng, F. S. Schlindwein, New approach for $\mathrm{t}$-wave peak detection and $\mathrm{t}$-wave end location in 12-lead paced ecg signals based on a mathematical model, Medical engineering \& physics 35 (8) (2013) 1105-1115.

[7] H. Zhu, J. Dong, An r-peak detection method based on peaks of shannon energy envelope, Biomedical Signal Processing and Control 8 (5) (2013) 466-474.

[8] Y. Sun, S. Suppappola, Nonlinear transforms of ecg signals for digital qrs detection: a quantitative analysis, Biomed. Eng. 41 (4) (1994) 397-400.

[9] H. Chatterjee, R. Gupta, M. Mitra, Real time p and t wave detection from ecg using fpga, Procedia Technol. 4 (2012) 840-844.

[10] K. Daskalov, I. Dotsinsky, I. Christov, Developments in ecg acquisition, preprocessing, parameter measurement and recording, IEEE Eng. Med. Biol. Mag. 17 (1998) 50-58.

[11] S. Bashir, A. D. Bakhshi, M. A. Maud, A template matched-filter based scheme for detection and estimation of t-wave alternans, Biomedical Signal Processing and Control 13 (2014) 247-261.

[12] M. Homaeinezhad, M. Erfanian, H. Naseri, A correlation analysis-based detection and delineation of ecg characteristic events using template waveforms extracted by ensemble averaging of clustered heart cycles, Comput. Biol. Med. 44 (1) (2014) 66-75.

[13] P. Addison, Wavelet transforms and the ecg:a review, Physiol. Meas. 26 (2005) R155-R199.

[14] C. Li, C. Zheng, C. Tai, Detection of ecg characteristic points using wavelet transforms, IEEE Trans. Biomed. Eng. 42 (1) (1995) 21-28.

[15] Z. Zidelmal, A. Amirou, M. Adnane, A. Belouchrani, Qrs detection based on wavelet coefficients, Comp. Met. and Prog. in Biomed. 107 (3) (2012) 490-496.

[16] J. Martinez, R. Almeida, S. Olmos, A. Rocha, P. Laguna, A wavelet-based ecg delineator: evaluation on standard databases, IEEE Trans. Biomed. Eng. 51 (4) (2004) 570-581.

[17] R. J. Martis, U. R. Acharya, L. C. Min, Ecg beat classification using pca, lda, ica and discrete wavelet transform, Biomedical Signal Processing and Control 8 (5) (2013) 437-448.

[18] A. Karimipour, M. Homaeinezhad, Real-time electrocardiogram p-qrs$\mathrm{t}$ detection-delineation algorithm based on quality-supported analysis of characteristic templates, Comput. Biol. Med. 52 (2014) 153-165.

[19] S. Banerjee, M. Mitra, Application of cross wavelet transform for ecg pattern analysis and classification, IEEE Trans. Ins. Meas. 63 (2) (2014)
326-333.

[20] http://physionet.org/physiobank/database/challenge/2011/set-a/.

[21] R. Mark, G. Moody, Mit-bih arrhythmia database, http://www.physionet.org/physiobank/database/mitdb/.

[22] S. Mallat, A Wavelet Tour of Signal Processing., Academic Press, 1999.

[23] M. Misiti, Y. Misiti, G. Oppenheim, J.-M. Poggi, Wavelets and their applications, ISTE Ltd, 2007.

[24] L. Senhadji, G. Carrault, J. Bellanger, G. Passariello, Comparing wavelet transforms for recognizing cardiac patterns, Engineering in Medicine and Biology Magazine, IEEE 14 (2) (1995) 167-173.

[25] S. Mahmoodabadi, A. Ahmadian, M. Abolhasani, Ecg feature extraction using daubechies wavelets, in: Proceedings of the fifth IASTED International conference on Visualization, Imaging and Image Processing, 2005, pp. 343-348.

[26] J. Serra, Image analysis and mathematical morphology, v. 1, Academic press, 1982

[27] J. P. Madeiro, P. C. Cortez, J. A. Marques, C. R. Seisdedos, C. R. Sobrinho, An innovative approach of qrs segmentation based on firstderivative, hilbert and wavelet transforms, Medical engineering $\&$ physics 34 (9) (2012) 1236-1246.

[28] Y.-C. Yeh, W.-J. Wang, Qrs complexes detection for ecg signal: The difference operation method, Computer methods and programs in biomedicine 91 (3) (2008) 245-254.

[29] M. S. Manikandan, K. Soman, A novel method for detecting r-peaks in electrocardiogram (ecg) signal, Biomedical Signal Processing and Control 7 (2) (2012) 118-128.

[30] R. Sameni, M. B. Shamsollahi, C. Jutten, G. D. Clifford, A nonlinear bayesian filtering framework for ecg denoising, Biomedical Engineering, IEEE Transactions on 54 (12) (2007) 2172-2185.

[31] R. Mitchell, Evaluation of adaptive line enhancement for beat-to-beat detection of ventricular late potentials, Electronics Letters 35 (13) (1999) 1037-1038.

[32] J. R. Hampton, The ECG made easy, Elsevier Health Sciences, 2013.

[33] Y.-C. Yeh, W. W-J., Qrs complexes detection for ecg signal: The difference operation method, Comput. Methods Prog. Biomed. 91 (2008) 245-254.

[34] S. M. Narayan, M. R. Franz, G. Lalani, J. Kim, A. Sastry, T-wave alternans, restitution of human action potential duration, and outcome, Journal of the American College of Cardiology 50 (25) (2007) 2385-2392.

[35] S. Danik, C. Cabo, C. Chiello, S. Kang, A. L. Wit, J. Coromilas, Correlation of repolarization of ventricular monophasic action potential with ecg in the murine heart, American Journal of Physiology-Heart and Circulatory Physiology 283 (1) (2002) H372-H381.

[36] R. Poli, C. S., G. Valli, Genetic design of optimum linear and nonlinear qrs detectors, IEEE Trans. Biomed. Eng. 42 (1995) 1137-1141.

[37] J. Madeiro, C. P.C., M. J.A.L, S. C.R.V, S. C.R.M.R, An innovatice approach of qrs segmentation based on first derivative, hilbert and wavelet transforms, Med. Engineering and Physics 34 (2012) 1236-1246.

[38] P. Hamilton, W. Tompkins, Quantitative investigation of qrs detection 
rules using the mit/bih arrhythmia database, IEEE Trans. Biomed. Eng. 33 (12) (1986) 1157-1165.

[39] A. Cabasson, O. Meste, G. Blain, S. Bermon, Estimation, analysis and comparison of the pr and rr intervals under exercise conditions and recovery, in: Computers in Cardiology, 2006, IEEE, 2006, pp. 497-500.

[40] A. Cabasson, O. Meste, G. Blain, S. Bermon, Quantifying the pr interval pattern during dynamic exercise and recovery, IEEE Transactions on Biomedical Engineering 56 (11) (2009) 2675-2683. 